\title{
Acceleration of the Solar Wind as a Result of the Reconnection of Open Magnetic Flux with Coronal Loops
}

\author{
L. A. Fisk ${ }^{1}$, G. Gloeckler ${ }^{1,2}$, T. H. Zurbuchen ${ }^{1}$, \\ J. Geiss ${ }^{3}$, and N. A. Schwadron ${ }^{4}$ \\ ${ }^{I}$ Department of Atmospheric, Oceanic, and Space Sciences, University of Michigan \\ 2455 Hayward St., Ann Arbor, MI 48109-2143, USA \\ ${ }^{2}$ Department of Physics and IPST, University of Maryland, College Park, MD 20742-4111, USA \\ ${ }^{3}$ International Space Science Institute, Hallerstrasse 6, CH-3012 Bern, Switzerland \\ ${ }^{4}$ Southwest Research Institute, San Antonio, TX 78228, USA
}

\begin{abstract}
There are compelling observations of a clear anti-correlation between solar wind flow speed and coronal electron temperature, as determined from solar wind ionic charge states. A simple theory is presented which can account for these observations, including the functional form of the correlation: Solar wind flow speed squared varies essentially linearly as the inverse of the coronal electron temperature. In this theory, magnetic field lines in the corona that open into the heliosphere reconnect with coronal loops near their base. This process displaces the open field line, and disturbs and imparts energy into the overlying corona, thereby determining the Poynting vector into the corona. This process releases mass from the loop into the corona, and determines the mass flux of the solar wind. The Poynting and mass flux into the corona determine the final speed of the solar wind, and yield a relationship that provides an excellent fit to observations.
\end{abstract}

\section{INTRODUCTION}

We present a simple theory to explain the compelling observations of Gloeckler et al. [1] of a clear anti-correlation between the solar wind flow speed and the coronal electron temperature, as determined from solar wind ionic charge states. The anti-correlation is consistent with a specific curve, motivated by the theory that is presented in this paper: Solar wind flow speed squared varies essentially linearly as the inverse of the coronal electron temperature.

The theory is an outgrowth of our work on the transport of open magnetic flux on the Sun $[2,3,4]$, and on the current understanding of the behavior of the coronal magnetic field and the development of coronal loops $[5,6,7]$. There is considerable evidence that open magnetic field lines on the Sun (those that open into the heliosphere) readily reconnect with closed magnetic loops. This results in a diffusive transport of the open flux that can account for the configuration of the heliospheric magnetic field, and offers an explanation for the formation of coronal holes and the apparent rotation of the large-scale current sheet that separates opposite polarities of the heliospheric magnetic field, during the solar cycle [4]. Processes similar to this are invoked to explain the evolution of the polar magnetic field of the Sun [8], and to explain the apparent ease with which the magnetic fields in coronal mass ejections (CMEs) become detached from the Sun, and do not result in a buildup of the magnetic field in the heliosphere [9]. 
The theory for the solar wind presented here is based on this simple process. An open field line reconnects with a closed magnetic loop and is displaced in its location. This displacement disturbs the overlying coronal magnetic field and deposits energy into the corona. The reconnection permits the mass originally stored on the coronal loop to be released onto the open field line. As we shall demonstrate with a set of remarkably simple assumptions, it is possible to derive a formula that exactly accounts for the observations of Gloeckler et al. [1].

We begin by reviewing the observations of Gloeckler et al. [1]. We then summarize the theory that can explain these observations. Full details of the theory can be found in Fisk [10]. Finally, we discuss some of the implications of this theory for other theories of the acceleration of the solar wind.

\section{THE OBSERVATIONS}

In Fig. 1, from Gloeckler et al. [1], time variations of the inverse of the coronal electron temperature $(1 / T)$ in units of $10^{6} \mathrm{~K}$ (open circles) and of the solar wind proton bulk speed $\left(V_{s w}\right)$ in units of $\mathrm{km} / \mathrm{s}$ (dotted curves) are plotted during a 166-day time period (August 27, 1996 - February 9, 1997) observed with SWICS on Ulysses. The coronal electron temperature is determined from the ratio of $\mathrm{O}^{7+} / \mathrm{O}^{6+}$, which freezes-in in the low corona. The data are 3-point running averages of the basic 12-hour averages. The tracking of the $1 / T$ and $V_{s w}$ curves is almost perfect except during the two time periods indicated by the shaded regions. Each of these two time periods coincides with a Coronal Mass Ejection (CME) event identified using bidirectional electron signatures in the Ulysses SWOOPS data (J. Gosling, private communication).

The relationship between solar wind speed and coronal electron temperature observed by Gloeckler et al. [1] is a surprise. There is no expectation that electrons in the corona have a major, direct role in the acceleration of the solar wind, particularly the fast solar wind. The temperatures and densities of the electrons, and the resulting pressure, are insufficient to accelerate the solar wind to the observed speeds of up to $\sim 800 \mathrm{~km} / \mathrm{s}$. This has led to numerous models for the acceleration of the solar wind in which the protons must obtain the required large pressures [e.g.,

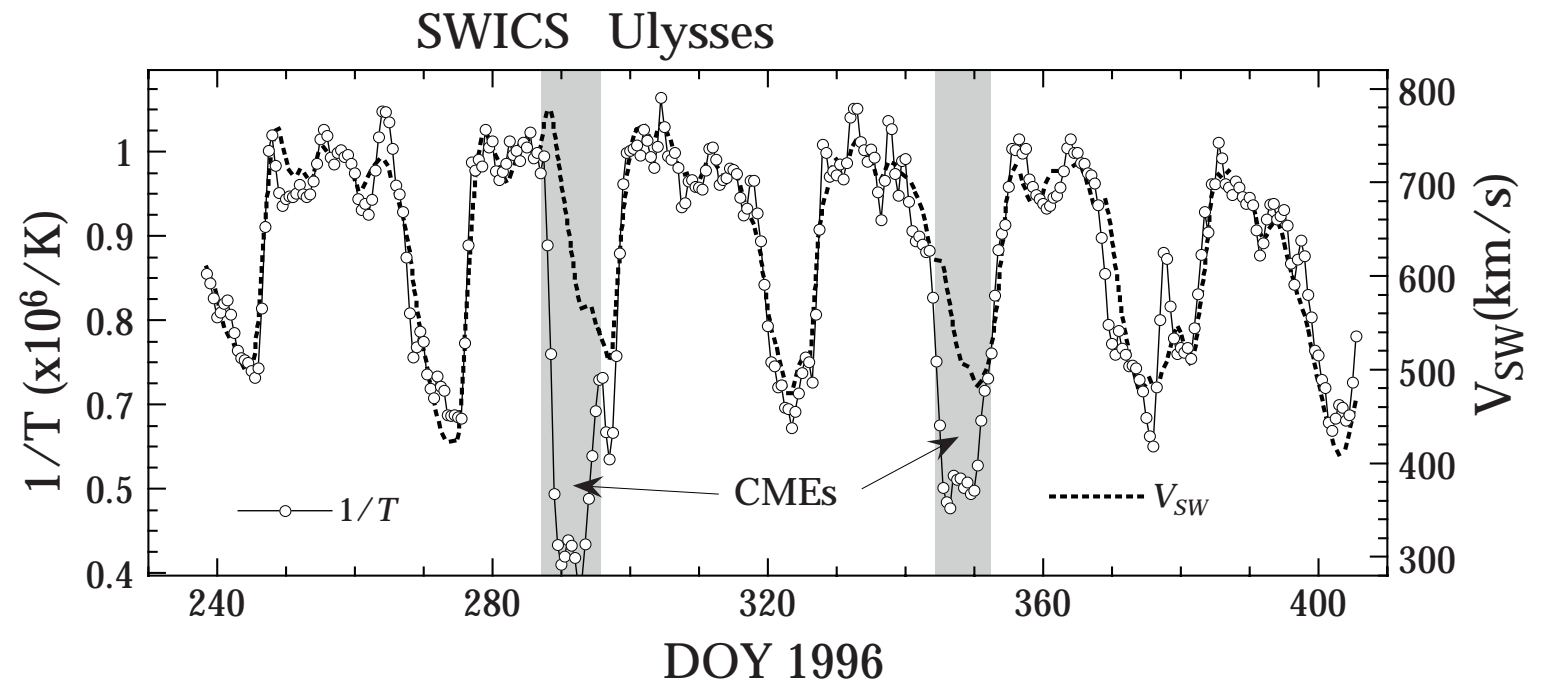

Figure 1. Time variations of the inverse of the electron temperature $(1 / T)$ in units of $10^{6} \mathrm{~K}$ (open circles) and of the solar wind proton bulk speed $\left(V_{s w}\right)$ in units of km/s (dotted curves) during a 166-day time period (August 27, 1996 - February 9, 1997) observed with SWICS on Ulysses. 


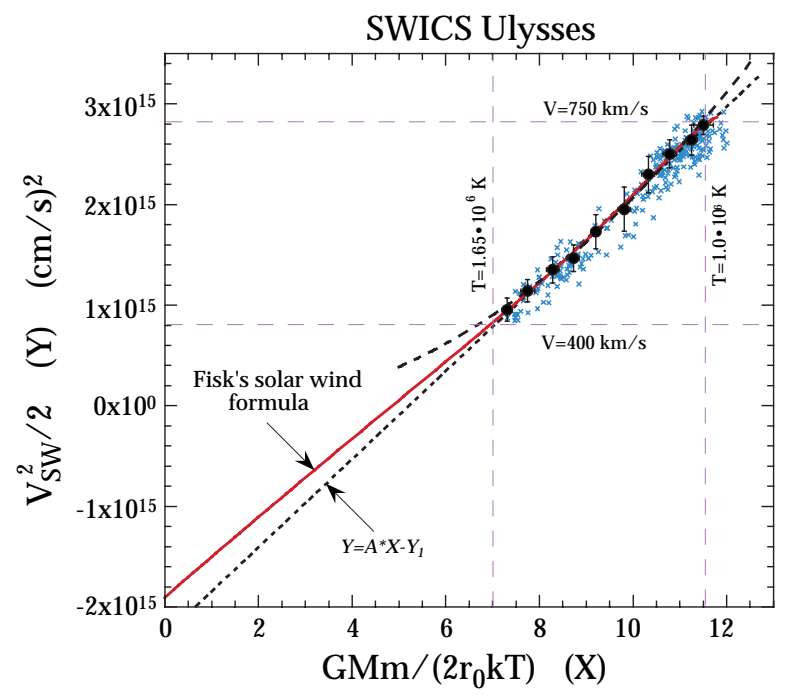

Figure 2. Scatter plot (gray crosses) of $Y=\left(V_{s w}{ }^{2}\right) / 2$ vs. $X=$ $\mathrm{GMm} /\left(2 \mathrm{r}_{0} \mathrm{k} T\right)$ using data of Figure 1 except for the two CME periods.

[11]]. It is perhaps equally surprising that the solar wind speed is anti-correlated with the coronal electron temperature. In models where there is both proton and electron heating, and yet the electrons remain cooler due to heat conduction into the chromosphere [11], we might expect that higher proton temperatures, and thus high flow speeds, were directly correlated with the coronal electron temperature. Rather, the observations of Gloeckler et al. [1], that flow speed and coronal electron temperature are anti-correlated, is providing us with unique information on conditions and processes occurring in the corona, which are in turn responsible for the final speed of the solar wind.

In Fig. 2, from Gloeckler et al. [1], a scatter plot (gray crosses) of $Y=\left(V_{s w}{ }^{2}\right) / 2$ vs. $X=$ $\mathrm{GMm} /\left(2 \mathrm{r}_{0} \mathrm{k} T\right)$ is created using data of Fig. 1 except for the two CME periods. The ten points indicated by solid circles are averages, and their error bars are standard deviations of $\left(X_{\mathrm{i}}, Y_{\mathrm{i}}\right)$ pairs binned in ten equal $X$ intervals.

There is, of course, scatter in the points, due to variations on the Sun and stream- stream interactions in the solar wind. Moreover, as would be expected, the specific curve, solar wind speed squared versus the inverse of the electron temperature, is most readily discernible when a broad range of solar wind speeds and coronal electron temperatures are considered. Such conditions are most prevalent at solar minimum, when both high and low speed flows occur. When a simple average is performed in these conditions, the specific curve holds for both fast solar wind from coronal holes and slower wind from elsewhere on the Sun. The only exception is solar wind plasma associated with CMEs, and even here, the relationship can be argued to hold with different choices for solar parameters [1].

\section{THE THEORY}

The theory for the solar wind model presented here is described in detail in Fisk [10]. The essential features of this model are based on a set of simple principles:

Magnetic loops are observed to occur everywhere on the Sun; they are believed to result from small bi-polar magnetic flux emerging through the solar surface and coalescing with each other by reconnection to form bigger loops (e.g. [6]), as is illustrated in Fig. 3. In coronal holes the loops are relatively small (heights $<15,000 \mathrm{~km}$ ) and cool $(<800,000 \mathrm{~K})$; outside of coronal holes the loops on the quiet Sun are larger (heights of $40,000-400,000 \mathrm{~km})$ and hotter $\left(\sim 1.5 \cdot 10^{6} \mathrm{~K}\right)$ [12].

Open field lines (magnetic field lines that open into the heliosphere) are present among the loops, in strong concentrations in coronal holes, but distributed also throughout the quiet Sun in lesser strength. 


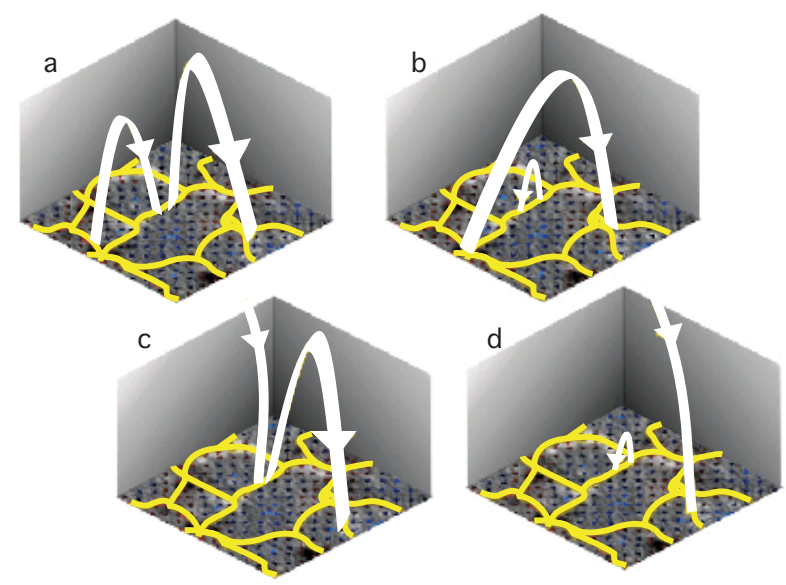

Figure 3. An illustration of the reconnection of loops and open field lines. In panel (a), the footpoints of two loops move with convective velocities along the lanes separating the granular and supergranular cells on the solar surface. In panel (b), two of the footpoints of the loops have reconnected to form a new larger loop and a small secondary loop that will subduct back into the photosphere. In panel (c), the footpoints of a loop and an open field line move along the lanes. In panel (d), a footpoint of the loop and the open field line have reconnected, the open field is displaced to lie over the location of another footpoint of the loop, and a small secondary loop is again formed that should subduct back into the photosphere.

As is illustrated in Fig. 3, an open field line can reconnect with the end of a loop with opposite magnetic polarity, presumably near the loop base, with three consequences: (a) The loop is effectively eliminated (a small secondary loop is created that appears to subduct back into the photosphere). (b) The open field line is displaced to lie over the location of the side of the original loop with the same polarity. (c) Mass is released from the loop onto the open field line.

The displacement of the open field line will disturb the overlying corona. Magnetic pressure variations will be introduced. When the coronal magnetic field relaxes back to equilibrium, work is done, and energy is assumed to be deposited in the corona in the form of heat. The amount of energy that is deposited can be readily calculated (see Fisk [10]).

The mass that is released through the reconnection process depends on the mass available in the loop. If the loops are iso- thermal, the available mass depends mainly on the scale height, which in turn depends on the isothermal temperature. For loop heights that are comparable to the scale height a small correction factor is required.

The energy that is supplied can be represented as a Poynting vector into the corona. Loops emerge through the solar surface, and thus represent an upward Poynting vector, for which there is no comparable downward Poynting vector since the loops are in large part eliminated by the reconnection with open field lines. The mass supplied through the reconnection process will determine the mass flux of the solar wind.

A simple energy balance equation can be used to specify the final solar wind speed squared, $V_{s w}^{2}$, in terms of the Poynting vector and mass flux [13]. It yields a unique formula (equation 11 of Fisk [10]) that predicts that the speed squared varies essentially linearly as the inverse of the loop temperature $T$,

$$
\begin{aligned}
& \frac{V_{S W}^{2}}{2}=\left(\frac{B_{\text {loop }}}{\rho_{\text {loop }}}\right)\left(\frac{\int \mathbf{B}_{\text {open }} \cdot d \mathbf{h}}{4 \pi r_{0}}\right)\left(\frac{G M_{0} m_{p}}{2 r_{0} k T}\right) \beta\left(h_{\text {loop }}, T\right)-\frac{G M_{0}}{r_{0}} \\
& \beta\left(h_{\text {loop }}, T\right)=\left\{1-\exp \left[-\left(1.75 h_{\text {loop }} G M_{0} m_{p}\right) /\left(2 r_{0}^{2} k T\right)\right]\right\}^{-1} .
\end{aligned}
$$

Here, $\mathrm{G}$ is the gravitational constant, $M_{0}$ is the mass and $r_{0}$ the radius of the Sun, $m$ is the proton mass, $\mathrm{k}$ the Boltzmann constant, and $h_{\text {loop }}$ is the height of the loop above the point of reconnection. $(B / \rho)_{\text {loop }}$ is the ratio of the magnetic field strength to the density of the loop at the base, where the reconnection with the open field line occurs and $\int \boldsymbol{B}_{\text {open }} \boldsymbol{\bullet} d \boldsymbol{h}$ is an integral along the open magnetic field from the surface of the Sun to large distances where $B_{\text {open }}$ becomes negligible. Fisk [10] points out that if the open magnetic field in the corona can be described as a potential field, this integral will be approximately constant for all open field lines, regardless of 
whether they undergo a radial or a superradial expansion, and has a value of $9.6 \times 10^{10} \mathrm{G}$.

The factor $\beta\left(h_{\text {loop }}, T\right)$ is significant only if the scale height and the loop height are comparable. For loops on the quiet Sun, Feldman et al. [12] find that the height of loops increases with increasing temperature of the material in the loops; hotter loops overlie cooler ones. Therefore, $\beta\left(h_{\text {loop }}, T\right)$ should be primarily a function of temperature but not a strong function of temperature since the temperature dependence enters as $h_{\text {loop }} / T$.

Thus, provided that the quantity $(B / \rho)_{\text {loop }}$ is relatively constant on the Sun and, $\beta\left(h_{\text {loop }}, T\right)$ depends only weakly on $T$, we predict that the acceleration of the solar wind depends on only one parameter, the temperature of the material in the originating loops, and that the final speed squared varies essentially linearly as $1 / T$. The quantity $(B / \rho)_{\text {loop }}$ would in fact be relatively constant, if loops expand such that the density and magnetic field strength stay proportional to one another.

The dependence on loop temperature in equation (1) arises simply because the mass available, and thus the mass flux, is proportional to the scale height, which in turn is proportional to temperature. The final speed squared of the solar wind varies inversely with the mass flux.

Equation (1) should hold in all forms of the solar wind: fast solar wind from coronal holes, where the loops involved are smaller and cooler; and slow solar wind from elsewhere on the Sun, where the loops are larger and hotter.

Equation (1) requires knowledge of the actual loop temperature. Gloeckler et al. [1] observe the solar wind ionic charge states and from that determine the electron temperature at the point in the corona where the charge states freeze-in. It is not unreasonable that these two temperatures will be nearly identical. First of all, the loops that are used are observed to have temperatures comparable to those inferred from solar wind charge states. A typical, relatively large coronal loop on the quiet Sun, which should be responsible for the slow solar wind, is observed to have temperatures $\sim 1.5 \cdot 10^{6} \mathrm{~K}[12]$, whereas coronal electron temperature inferred from charge states is $\sim 1.7 \cdot 10^{6} \mathrm{~K}[14]$. Similarly, in the fast solar wind, the loops have temperatures $\sim 800,000 \mathrm{~K}$, whereas the temperature inferred from charge states is $\sim 1.0 \cdot 10^{6} \mathrm{~K}$. These apparent systematic differences could result from the reconnection process itself, when the open field line reconnects with the loop. A small amount of heat could be imparted to the loop. The actual release process itself, in which there is a sudden drop in density, could facilitate the freeze-in of ionic charge states at the point of release. Conversely, the free flow of electrons along the open field lines could preserve the electron temperature near the loop value, and the freeze-in occurs at the more traditional distances of a few solar radii [e.g. 15,16]. In the detailed numerical model of Schwadron [17], the calculated solar wind charge states are found to be representative of the electron temperatures in the loops. Protons, in contrast, need to be heated in the corona by the dissipation of the energy imparted by the displaced open field lines, in order to form the solar wind.

All that is required to test equation (1) using SWICS observations is that there is a oneto-one relationship between the coronal electron temperature inferred from charge state measurements and the actual temperature of electrons in the loop. For example, if these two temperatures differ by a constant amount, there is no change in the use of equation (1) to relate observed solar speed to freezing-in temperature from observed charge states, other than a small adjustment to the inferred dependence of loop heights on electron temperature in that loop. 


\section{COMPARISON WITH OBSERVATIONS}

The dotted curve in Fig. 2 is a linear leastsquares fit to the data, the dashed curve is a linear function, speed vs. $1 / T$, and the solid curve is a fit using equation (1). Clearly, (1) does provide an excellent fit to the data. It is necessary in obtaining the detailed fit to have loop height vary directly with loop temperature. Note that the intercept of the curve, $G M_{o} / r_{o}$, the gravitational potential per unit mass of the Sun, is not an adjustable parameter.

\section{CONCLUDING REMARKS}

There are, of course, many solar wind theories more complicated than the one presented here [18, 19 and references therein; 20 and references therein; 21, 22, 23, 24]. No doubt, in time, some of these complexities will have to be added to our simple theory, and more completeness achieved. Nonetheless, the simple points made here do account for the observations, and, indeed, the challenge perhaps is to other theories. The clear anti-correlation between solar wind speed and coronal electron temperature of Gloeckler et al. [1], and the apparent simple relationship between these two quantities, represent a critical test against which all solar wind theories should be judged.

\section{ACKNOWLEDGMENTS}

This work was supported, in part, by NASA grant NAG5-10975, NSF grant NSF0096664, NASA/ JPL contract 955460, and NASA/CalTech contract NACG5-6912.

\section{REFERENCES}

1. Gloeckler, G., Zurbuchen, T. H., and Geiss, J., J. Geophys. Res., in press (2002).

2. Fisk, L. A., J. Geophys. Res. 101, 15,549 (1996).

3. Fisk, L. A., Zurbuchen, T. H., and Schwadron, N. A., Astrophys. J. 521, 86 (1999).

4. Fisk, L. A., and Schwadron, N. A., Astrophys. J. 560, 425 (2001).

5. Schrijver, C. J., Title, A. M., van Ballegooijen, A. A. H., Hagenaar, J., and Shine, R. A., Astrophys. J. 487, 424 (1997).

6. Handy, B. N., and Schrijver, C. J., Astrophys. J. 547, 1100 (2001).

7. Simon, G. W., Title, A. M., and Weiss, N. O., Astrophys. J. 561, 427 (2001).

8. Schrijver, C. J., DeRosa, M. L., and Title, A. M., Astrophys. J., in press (2002).

9. Crooker, N., Gosling, J. T., and Kahler, S. W., J. Geophys. Res. 107, 148 (2002).

10. Fisk, L. A., J. Geophys. Res., in press (2002).

11. Hansteen, V. H., Leer, E., and Holzer, T. E., in Solar Wind Nine, edited by S. R. Habbal et al., AIP Conference Proc. 471, Melville, New York, 1999, p. 17.

12. Feldman, U., Widing, K. G., and Warren, H. P., Astrophys. J. 522, 1133 (1999).

13. Fisk, L. A., Schwadron, N. A., and Zurbuchen, T. H., J. Geophys. Res. 104, 19,765 (1999).

14. Von Steiger, R., Schwadron, N. A., Fisk, L. A., Geiss, J., Gloeckler, G., Hefti, S., Wilken, B., WimmerSchweingruber, R. F., and Zurbuchen, T. H., J. Geophys. Res. 105, 27,217 (2000).

15. Burgi A., and Geiss, J., Solar Physics 103, 447 (1986).

16. Geiss, J., Gloeckler, G., von Steiger, R., Balsiger, H., Fisk, L. A., Galvin, A. B., Ipavich, F. M., Livi, S., McKenzie, J. F., Ogilvie, K. W., and Wilken, B., Science 268, 1033 (1995).

17. Schwadron, N. A., J. Geophys. Res., submitted (2002).

18. Parker, E. N., Astrophys. J. 128, 664 (1958).

19. Isenberg, P. A., The solar wind, in Geomagnetism, vol. 4, edited by J. A. Jacobs, Academic Press, Orlando, 1991, p. 1.

20. Marsch, E., Adv. Space Res. 14, 103 (1994).

21. Hansteen, V. H., and E. Leer, J. Geophys. Res., 11, 21,577 (1995).

22. Axford, W. I., and McKenzie, J. F., "The solar wind," in Cosmic Winds in the Heliosphere, edited by J. R. Jokipii, C. P. Sonett, and M. S. Giampapa, University of Arizona Press, Tucson, 1997, p. 31.

23. Hollweg, J. V., J. Geophys. Res. 105, 15,699 (2000).

24. McKenzie, J. F., Axford, W. I., and Banaszkiewicz, M., Geophys. Res. Lett. 24, 2877 (1997). 